fundamental studies of physical changes in the structure of surfaces when they are deformed by abrasion or other processes.

The manufacture of almost all metal articles involves plastic deformation of the metal. The Plasticity Division's work is directed towards providing a better understanding of the mechanism of plastic deformation so that economies in plant design and more efficient operation may be achieved. When $\alpha$-uranium is irradiated with neutrons a greatly accelerated creep takes place, even under small loads. The mathematical theory of plasticity has been applied to explain, in detail, the mechanism of this phenomenon in a uranium aggregate, starting from the known crystallographic properties of a single crystal of uranium.
In many metal-working processes the deformation takes place in the presence of hydrostatic pressure which may be considerably greater than the yield stress of the metal. Equipment has been developed for carrying out simple mechanical tests under pressure up to 100 tons per square inch. Tensile tests on various materials under a range of hydrostatic pressures have shown that there is, generally, a marked increase in their ductility as the pressure is increased. With copper, aluminium and cast-iron the increase in ductility is gradual, with zinc and 'Mazak' (a zinc-base alloy) there is an abrupt change from brittle to ductile behaviour for a small change of pressure. The possibility of extruding brittle materials by carrying out the process under high pressure is being examined. R. J. F. FrANkLIN

\title{
ACCURATE MEASUREMENT OF THE VISCOSITY OF LIQUIDS
}

A SYMPOSIUM on the "Accurate Measurement of the Viscosity of Liquids", arranged jointly by the Institute of Petroleum and the British Society of Rheology, was held on June 6 at the Institute of Petroleum, 61 New Cavendish St., London, W.1. It was under the chairmanship of Mr. W. Pohl (chairman of the Lubrication Committee, Institute of Petroleum) and Prof. E. G. Richardson (University of Durham, president of the British Society of Rheology).

The revised standard methods (British Standard $188: 57$ and Institute of Petroleum, $71: 58$ ) for measuring the viscosity of liquids in C.G.s. units, which incorporate the experience and the results of co-operative experimental work obtained in Britain and other countries during the post-war years, describe a 'stepup' procedure for the calibration of glass capillary-type viscometers, using master viscometers and freshly distilled water at $20 \cdot 00^{\circ} \mathrm{C}$. with an agreed viscosity of 1.002 centipoises ( 1.0038 centistokes). Dr. R. Roscoe (University of Durham) pointed out in a paper on the determination of viscosity by the oscillating vessel method that further research on accurate absolute methods of viscosity measurement of liquids as standards is desirable, because of the difficulty in the capillary tube method of the elimination of the end correction. The slight inconsistencies in the careful measurements of J. F. Swindells, J. R. Coe and T. B. Godfrey, who found the viscosity of water at $20.00^{\circ} \mathrm{C}$. to be $1.0019 \pm 0.0003 \mathrm{cP}$., arise from small differences in the ends of paired tubes. The oscillating vessel method, advocated by Prof. Andrade and A. Chiong, may prove to be better for accurate viscosity measurements. Dr. Roscoe described a series of precise measurements with water, using oscillating vessels, both spherical and cylindrical, supported by a graduated capillary tube so that the water acted as its own thermometer. In this preliminary work a value obtained for the coefficient of viscosity for water at $20^{\circ} \mathrm{C}$. was $1.0025 \pm 0.0005$, but an analysis of the sources of error in the results suggests that with fairly simple improvements in technique, for example, using cylindrical vessels and measuring angular velocity rather than amplitude of oscillation, the accuracy of the method, of the order one part in 10,000 , could be substantially greater than that obtained in capillary tube measurements.

The measurement of viscosity by means of a vibrating system using an electronic counting technique was described by Mr. M. T. Browne (Imperial College and Queen Elizabeth College, London), who gave the method of calculating viscosity values for water from the logarithmic decrement, the electronic apparatus counting the number of cycles of vibration occurring while the amplitude decreases from one value to half this value.

For oils of higher viscosity the liquid is located on a plane end of the rod. The apparatus is particularly adapted to measure small changes of viscosity.

In a paper on the precise measurement of kinematic viscosity in the petroleum industry, K. A. Lammiman and P. P. Morris (Esso Research Ltd.) described standard instruments and modern equipment involving electronic timing devices, precisely controlled thermostat baths for a temperature-range from $-100^{\circ}$ to $200^{\circ} \mathrm{C}$. and above, and the technique used for the scrupulous cleaning of instruments by pressure jets of solvents. The authors described methods of measuring viscosity when only small amounts were available; these required the use of the standard miniature British Standard viscometers, torque viscometers such as the plate-cone type, vibrating systems and a comparative method of the rate of descent of an index of oil $(0.05 \mathrm{ml}$.) in an inclined capillary.

Methods of examining opaque oils and fuel oils and their heat pretreatment were also described, as well as semi-automatic methods using viscometers fitted with platinum probes at the fiduciary markings.

During the past two decades industry has become 'shear' conscious mainly in connexion with the requirements of the Armed Services and the automotive industry. At the rates of shear occurring during flow in standard viscometers, the viscosity does not show variations, but at high rates of shear the coefficient of viscosity is no longer a constant. Dr. $R$. Schnurmann (Esso Research Ltd.) described a jet viscometer in which frictional heating is practically eliminated when a liquid is forced slowly and in individual drops through a narrow streamlined constriction.

By varying the pressure difference across the aperture or the temperature the rate of shear can be altered over a wide range. The kinetic energy correction can be determined experimentally and is smaller by a factor of 2 than for long capillary viscometers, which usually have a length to diameter ratio of 100 . 
The viscosity of any liquid containing asymmetric molecules can be made to show a temporary viscosityfall during flow because of molecular orientation in the flow stream. Castor oil, for example, appears to have a constant viscosity coefficient for rates of shear up to $5,000 \mathrm{sec}^{-1}$ yet at a rate of shear of 250,000 sec. ${ }^{-1}$ its viscosity is reduced by about 25 per cent. The apparent viscosity over a wide range of rate of shear has a falling characteristic with a point of inflexion, the curve bending upward when an appreciable proportion of the pressure-volume energy is converted into kinetic energy and when turbulent flow takes place. The viscosity of a liquid containing large asymmetric molecules, as in an oil thickened with polymer, which has suffered a permanent viscosity loss through severe shearing, is more sensitive to temperature change than that of the liquid at low rates of shear. On the other hand, when a liquid suffers a temporary viscosity reduction its viscositytemperature coefficient is smaller while a shearing stress is being applied than when the stress has been removed.

The complexity of the flow behaviour of solutions of some polystyrenes at high dilution was emphasized by Dr. P. P. Rutherford (University College of North Staffordshire), who showed that with a capillary-type viscometer anomalous $\eta s p / c$ and $\ln \eta r / c$ values were obtained at the highest dilutions. Upswings in the graphs were observed when these values were plotted against the concentration over the range $0 \cdot 2-0 \cdot 4 \mathrm{gm}$. of polystyrene (mol. weight less than 40,000). The concentration at which these upswings occurred depends on the intrinsic viscosity or molecular weight, the temperature and the kind of solvent used. The flow times for the dilute solutions left in contact with powdered glass for several months were found to be almost unaltered, indicating that adsorption effects on the viscometer walls are negligible. Before an explanation of the anomalies is offered further work is necessary not only on viscosity measurements of fractionated polystyrenes of low molecular weight but also by the measurement of light scattering, density and other properties in the low concentration range.

At the first World Petroleum Congress held at the Imperial College of Science and Technology, 1933, the opinion was expressed by Dr. Guy Barr of the National Physical Laboratory that the international standardization of viscosities should be made with viscometers of known characteristics, as these are much less likely to change than standard viscosity oils.
This view is at present maintained by both the British Standards and Institute of Petroleum viscosity committees, and in a short report by Mr. W. O. Jennings (National Physical Laboratory) and Dr. E. W. J. Mardles (chairman of the Institute of Petroleum Viscosity Panel) it was shown by results obtained at the National Physical Laboratory that the average change in the constant of four master viscometers over the period 1953-58 was no greater than +0.01 per cent.

Information from observation books at the National Physical Laboratory indicated the satisfactory working of the standard methods. The report using these results gives the method of establishing a series of reference viscometers on the basis of the agreed viscosity value of water. The accuracy of the method was assessed by cross checking with other laboratories.

The meeting discussed the various factors by which high precision is attainable and in particular the difficulty of using the quadratic equation, $\nu=C t-$ $B / t$ where $\nu$ is kinematic viscosity, $C$ the viscometer constant, $t$ time of flow and $B$ the coefficient of the kinetic energy of efflux, $B$ being known to be variable. The standard methods indicate ways and means of avoiding the use of $B$ or obtaining a working value for it by calculation.

The experimental determination of term $B$ can conveniently be made, as shown in the report, by first obtaining the value for $C$, using a flow time not less than 1,200 sec.; then, with a flow time of about, but not less than, 200 sec., evaluating $B$ from the equation $B=C t^{2}-v t$, where $t$ is the short flow time and $C$ the constant already obtained.

L. A. Steiner (Viscosity Panel, Institute of Petroleum) described a series of experiments showing the variation of the coefficient $B$ under different conditions of experiment. Using sets of viscometers of the same design but with one variant, namely the capillary diameter, the true constants could be ascertained by a graphical method. It was shown that the variation in the coefficient $B$ could be explained partly by end effects, the shapes of the entry and exit ends of the capillary affecting the results; part of the efflux energy is recovered during the filling of the bulb.

The meeting included the exhibition of some new viscometers and equipment, and was concluded by a visit to the viscometry, chronometry and thermometry sections of the National Physical Laboratory on June 7.
E. W. J. MardLES

\section{THE BRITISH GELATINE AND GLUE RESEARCH ASSOCIATION}

$T$ HE fifteenth meeting of the Research Panel of the Association was held on June 12, with Dr. G. R. Tristram (University of St. Andrews) in the chair. The meeting was well attended and was followed by demonstrations and discussions in the Association's Laboratories at $2 a$ Dalmeny Avenue, London, N.7.

The meeting commenced with a review by Prof. A. Neuberger (St. Mary's Hospital Medical School, London), with the title "General Aspects of the Metabolism of Collagen". Prof. Neuberger outlined the development of the use of radioactive amino-acids in studies of the laying down and turnover of collagen, and the part which the soluble collagens play in this. He also described work showing the inability of an animal to make use of free hydroxyproline (and hydroxylysine) in collagen synthesis, the hydroxyproline being formed at some stage by the hydroxylation of bound proline.

Drs. N. M. Green and D. A. Lowther (St. Mary's Hospital Medical School) gave a paper "Biosynthesis of Collagen", describing their recent researches. In these they have made use of tissue slices obtained from the connective tissue tumour formed when carrageenin is injected under the skin of a guinea pig. Two problems were studied : the first, the conversion of proline into collagen-bound hydroxyproline, and secondly, the precursor relationships 